they perform at their best while everything is held together as they do so. Such leadership relies on the power that comes from garnering information (with the ears as well as the computer terminal) and using it to analyse and persuade. It relies on understanding and influencing those who wield executive, political, or professional power, working alongside them, recognising or renegotiating their domains, and breaking down barriers of mutual suspicion. It will rely too on keeping a clear vision of what remains to be achieved. ${ }^{+6}$

Many have doubted whether there will be sufficient resources to implement the Acheson report or enough trained staff to fill the new posts of district controllers of communicable diseases. It would be a foolish repeat of past mistakes to expect the new directors of public health to deliver the goods without having the means to produce them. The task of reviewing training in the basic sciences of public health is already beginning. Few, however, have recognised how much the skills of leadership will be fundamental to applying those sciences. Such skills are rare and will not magically appear. They must be developed, learnt, and constantly improved on, and training programmes need to be designed imaginatively.
In short, public health is too important for its practitioners to succumb to the old syndrome of chronic identity crises compounded by organisational impotence. ${ }^{*}$ The people's health is-or should be-the concern of a wide and mixed range of people and organisations. Those now charged with the role of coordinating these groups have a huge job to do, and public health physicians face a change as radical as that which surgeons underwent a century ago. We must meet that challenge by developing new and appropriate skills, not by reinventing the old public health.

Senior Registrar in Community Medicine,

Parkside Health Authority,

London W2 INY

Cunningham D. Public health in London. Br.Med 7 1988:297:65

2 Committec of Inyuiry Into the Future Develonment of the Public Health Function. Public health in Eingland. London: HMSO, 1988. Acheson report

Ruc R. Advising a health authority. The Community P' Pvicion 1987:2:3.

t Harvev-Jones J. Making it happen: reflections on leadership. London: Collins, 1988.

Turrill EA. Change and innovation: a challenge for the NHS. London: IHSM. 1986.

6 Kotter JP, Lawrence JP. Mavors in action. Five studies in urgan gocemance. New York: John Wiley Kotter

7 Binstead D. Decelopments in interpersonal skills training. London: Gower, 1986.

8 Lewis J. What price community medicine? The philosophy, practice and politis of public health since 1919. Brighton: Wheatsheaf Books, 1986

\title{
What went wrong at Exeter?
}

\section{Many patients overexposed to radiation but only a few at serious risk}

The recent news that 153 patients with cancer had been given doses of radiation $25 \%$ greater than intended has shocked departments of radiotherapy and oncology across the country. This is the worst radiotherapy dosimetric error ever experienced in the United Kingdom. On the face of it, it seems to have been due to simple miscalculation of dose output by a medical physicist after the installation of a new radioactive cobalt source, a standard procedure carried out every three to four years in all departments using telecobalt equipment.'

The new source, which did not come directly from Amersham International (the usual supplier) because it had previously been used in another centre, arrived in Exeter in February of this year. The dose excess seems to have been due to a mistake in measuring the time over which the reference dose was calculated. The physicist may have multiplied by the wrong factor to achieve an equivalent exposure for one full minute. Tragically, this inaccuracy was not then recognised, possibly because the physicist was working on his own and his figures may not have been checked. Although there are no mandatory guidelines relating to checking, the initial calibration of a new highly active cobalt source is the most fundamental dose calibration during the life of that part of the equipment, and subsequent calibration exercises would be based on this figure, making additional inaccuracies more difficult to recognise later on.

What safeguards would normally apply after the installation of a new cobalt source? The most important is that the suppliers also include a test certificate, normally sent with the new source itself, which should be returned after installation and calibration with the physicist's calculation of the dose, usually in roentgen/min at 1 metre $(\mathrm{Rmm})$. Perhaps this would be better if done "blind," but Amersham would normally send its expected figure on the certificate, and in our experience at University College Hospital over the past six source changes our own recording of the absorbed dose in $\mathrm{Rmm}$ has invariably been within $2 \%$ of the expected level. Was this normal procedure carried out at Exeter?
A further uncertainty surrounds the question of how the error was finally noticed. Despite press reports that the vigilance of nursing and radiographic staff led to unexpected skin reactions arousing suspicion, it seems equally possible that the five month period of overexposure (February to July) was brought to an end by chance. A radiation "phantom" apparatus, designed to test absorbed radiation dose within biologically equivalent tissues, was being used in a multicentre comparison of dosimetric consistency, organised by the Institute of Physical Sciences in Medicine. The phantom reached Exeter in July, and unexpectedly high readings gave the game away. Ironically, despite a request by the institute to the Department of Health and Social Security for funding for this project, the department decided that it was not sufficiently important or likely to prove of practical benefit. It would be very disturbing if neither the doctors, the nurses, nor the radiographers concerned picked up a consistent excess in the radiation dose of $25 \%$ over several months, and many radiotherapists would agree that an excess of $15 \%$ or so would normally be clinically detectable. Before we reach a hasty conclusion it is well to remember that the department of radiotherapy and oncology at the Royal Devon and Exeter Hospital has a high reputation for excellence and has, indeed, pioneered the use of new radiotherapy techniques now finding general application. Its throughput is substantial, over 1700 new cases a year, spread among three consultants, who themselves see patients throughout the whole of Devon and are therefore necessarily off site for part of the working day. Effectively there are no junior staff experienced in radiotherapy. Similarly, the department of radiation physics is also overstretched and split between two sites. By the standards of our European or North American colleagues, all British departments of radiotherapy and oncology are understaffed, and most radiotherapists in the United Kingdom see more patients each year than is recommended by the Royal College of Radiologists.

In practice, all radiotherapists know that their own 
approach may differ from that of their colleagues, often to a surprising degree, particularly in dose and fractionation. In the south of England, for instance, a radical course of therapy may take six weeks, whereas colleagues at the Christie Hospital in Manchester, the largest radiotherapy centre in Europe, would hardly ever treat a patient for longer than three weeks. For a given clinical problem, therefore, the overall dose of radiotherapy might well vary by a factor of at least $25 \%$. Furthermore, refinements in technique may lead within a relatively short time to revisions in dosage; for treast conserving carcinoma of the breast, for example, the radiation dose given by one of the Exeter consultants still remains lower than the dose he would have given under similar circumstances five years ago, even including the unintentional $25 \%$ excess.

None the less, a serious error has been made, though only a small minority of patients are at serious risk of damage. Patients who were treated palliatively, many of whom had widespread metastases, are unlikely to survive long enough to suffer severe damage; in any event palliative doses are often low so a $25 \%$ additional dose would be relatively harmless.

Professor Charles Joslin from the University of Leeds has already started an independent inquiry into this accident, and his findings will be made available to the regional health authority. It already seems clear that a small number of patients are indeed at high risk of radiation damage, which could be extremely serious-for example, laryngeal necrosis, rectal stenosis, and fistula. In a recent high court case a claim for damages was made against a health authority after a laryngectomy was performed for laryngeal radionecrosis, necessitated by a course of treatment in which a similar error of dose calculation led to an unexpectedly high dose.

One clear lesson from this unfortunate business is that calibration of a new cobalt source must be checked and rechecked, with better communication between the hospital physicist and the suppliers, who should have an accurate record of the likely output of the source to be installed. It is certainly possible to cross check a new installation in this way, and it might even be sensible to repeat the calibration of a new source a month after its first use in case of contamination with other isotopes which might have unexpected patterns of decay. There will also be far reaching consequences for the Hospital Physicists' Association, which is properly arguing for increased status for medical physicists and may now have to turn its attention to the question of insurance and indemnity for its members.

Consultant in Radiotherapy and Oncology,

JEFFREY S TOBIAS

University College Hospital,

London WC1E 6AU

1 National Radiological Protection Board. (iuddance notes for the protectum of persoms aguinst iomsing radiutions arising from medical and dental use. London: HMSO, 1988.

\title{
Social network interaction: new jargon in health inequalities
}

\author{
Whom you know may be important for your health
}

Gomer and Johnson have recently described the relations between social network interaction and mortality.' This horrible piece of jargon has grown out of the increasing evidence on psychosocial factors and their effects on health. The phrase should not be forgotten because it seems important in explaining patterns of mortality.

Interest in differences in mortality among social groups has re-emerged in the past 10 years both internationally ${ }^{2}$ and nationally. ${ }^{3}$ This follows a long period when it was assumed in most developed countries that reorganisation of health and social welfare provision after the second world war had reduced or even eliminated the major differences among the social groups and the discovery in the late 1960s that this was not the case. ${ }^{+}$Differences in mortality among social groups were expected to decline because the poorest were protected by administrative changes. It was assumed that socioeconomic inequalities had declined and that the general rise in living standards among young people had virtually eliminated the so called "diseases of poverty."

Interest in inequalities re-emerged at a time when it seemed that the major improvements in health in the early part of the century were slowing down and even stopping. Infectious diseases, particularly tuberculosis and respiratory disease, were examples of causes of death with strong links to poverty, whose importance had obviously diminished. It came as a surprise, then, when in the 1960 s and 1970s many causes of death that had not previously shown any tendency to be related to social class started to develop the now widely accepted pattern of low mortality among professionals and high mortality among semiskilled and unskilled manual workers. ${ }^{5}$

The pattern of social inequality in health that has emerged in recent years has led to greater emphasis being placed on explanations and on the search for factors that may exert a more general influence on health but which are further back in the causal chain. ${ }^{6}$ Since 1980 there has been limited evidence that these inequalities are an artefact of the method used to produce the official statistics and that differences are caused by health related mobility among social classes (the social selection hypothesis). These explanations have, however, been measured and shown to be unimportant.

As Syme suggested, social epidemiologists should investigate "ways in which a person's position in the social structure influences the likelihood that he will develop disease." This includes differences in resistance and response to the social environment as well as differences in environmental stressors." Cassel asked, "Are there categories or classes of environmental factors that are capable of changing human resistance in important ways and making subsets of people more or less susceptible to these ubiquitous agents in our environment?" $\mathrm{He}$ went on to argue that "the presence of other members of the same species, or, more generally, certain aspects of the social environment would be one such category of environmental factor." 10

\section{Resources for coping}

Valkonen claims that interest in general factors has been stimulated not just by the pattern of mortality differences among social classes now being observed for most causes of death. Another reason is that studies such as the Whitehall study fail to explain with known risk factors a large fraction of differences that are observed in particular causes of death such as coronary heart disease. ${ }^{21}$ In the search for general 